\title{
Design of Polarization-Dependent Reflectarray for Terahertz Waves
}

\author{
Tiaoming Niu*†, Withawat Withayachumnankul*, Derek Abbott*, and Christophe Fumeaux* \\ ${ }^{*}$ School of Electrical \& Electronic Engineering, The University of Adelaide, Adelaide, SA 5005, Australia \\ ${ }^{\dagger}$ School of Information Science and Engineering, Lanzhou University, Lanzhou 730000, P. R. China \\ Email: niutm@eleceng.adelaide.edu.au
}

\begin{abstract}
A reflectarray is designed for polarizationdependent beam deflection at 1 THz. Each unit cell is composed of two sets of dipole resonators, corresponding to orthogonal polarizations. The orthogonal dipoles are arranged in two interlaced triangular-lattice arrays. One subarray containing multiple unit cells provides a gradient phase response to complete a $2 \pi$ phase cycle over its length. This design demonstrates that it is possible to separate the polarization components of an incident beam by deflecting them into two different directions in a given plane. Numerical results predict successful implementation of the principle with available materials and within realistic manufacturing tolerances for operation at a frequency of $1 \mathrm{THz}$.
\end{abstract}

\section{INTRODUCTION}

The reflectarray concept has been adopted across the electromagnetic spectrum from the microwave range to optics [1][5]. Its function of accurately shaping reflected beam characteristics plays an important role for specific applications in wireless communications. By tailoring the geometrical sizes of the resonators that compose the discretized reflecting surface, one can freely engineer the phase response on the surface of the reflectarray to achieve the required field distribution for scattered waves [6]. A similar concept has been adopted to realize efficient transmitarrays [7], [8]. For the reflectarrays, radiating resonant elements of various shapes on substrates with different materials have been implemented. Patches and strip dipoles are traditional radiating elements that have been widely used at microwave frequencies [9]. In recent years, some tunable materials, such as graphene [10] and liquid crystal [11]-[13], have also been discussed for designing reflectarrays, particularly for more versatile performance towards higher frequencies.

Realization of a dual-polarization reflectarray has gained attention due to its ability for discriminating incident polarizations. Multilayer elements with rectangular patches [14], and cross-shaped microstrip loops combined with PIN diodes [15] have been used as unit cells for constructing dual-polarization reflectarrays in the microwave regime. However, in the terahertz and optical ranges, such stacked multilayer structures are challenging to manufacture with required precision. In this article, by using strip dipoles in an interlaced triangular-lattice arrangement, we propose a polarization-dependent terahertz reflectarray, i.e., with different polarizations deflected into different directions.

\section{UNIT CELL RESPONSE}

As shown in Fig. 1, a unit cell is composed of two sets of orthogonal dipole resonators, each set corresponding to a particular polarization. A configuration of two interlaced triangular-lattice arrays is chosen to reduce mutual coupling [16]. The unit cell comprises three layers: dipoles made of gold as the top layer, a PDMS dielectric spacer as a substrate and a platinum ground plane. For the metals, the material parameters used in [17], i.e. surface impedance $Z_{A u}=0.287+j 0.335 \Omega$ for gold and $Z_{P t}=0.628+j 0.667 \Omega$ for platinum, are adopted here, while the relative permittivity 2.35 and loss tangent 0.03 of PDMS are determined from measurement [18]. For operation at $1 \mathrm{THz}$, the size of the unit cell and the thickness of the PDMS substrate are selected at fixed values $2 a=300 \mu \mathrm{m}$ and $h=20 \mu \mathrm{m}$, respectively. Different phase responses for a particular polarization can be achieved by varying the length and width of a corresponding dipole. A phase response profile is simulated by using uniform infinite arrays in Ansys HFSS commercial software with master-slave boundary conditions. Based on a numerical analysis, it is confirmed that the effect of a dipole is negligible for the incident wave with polarization orthogonal to its longitudinal axis. Therefore, in the optimization of the unit cell shown in Fig. 1, the dipoles perpendicular to a given polarization are fixed, while the length and width of the dipoles parallel to the polarization are varied. In addition, in order to test the effect of the substrate loss on the reflection coefficients, three values of loss tangent, $\tan \delta=0,0.03,0.06$, are applied in the simulation. The numerically resolved reflection phase and magnitude responses as a function of the dipole length for an infinite uniform array are given in Fig. 2(a) and (b), respectively. From the simulation results, a phase range of over $300^{\circ}$ is obtained, which is sufficient for designing a periodic gradient reflectarray if the progressive phase change is larger than $60^{\circ}$. It is obvious that the loss of the substrate can significantly affect the reflection coefficient for the designed structure. A larger loss tangent leads to a sharper phase change and higher loss, and therefore the performance of the designed reflectarray would be more sensitive to the tolerance in the dimensions.

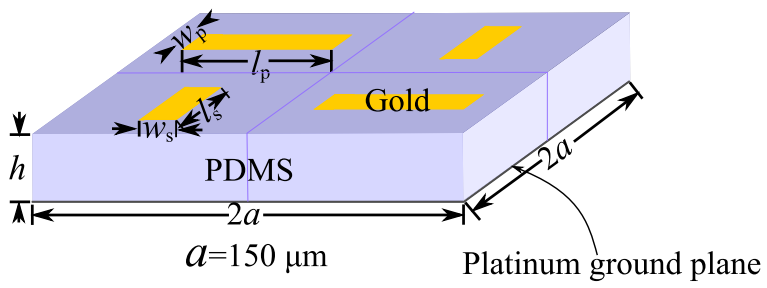

Fig. 1. A unit cell containing four dipoles for the polarization-dependent reflectarray. 

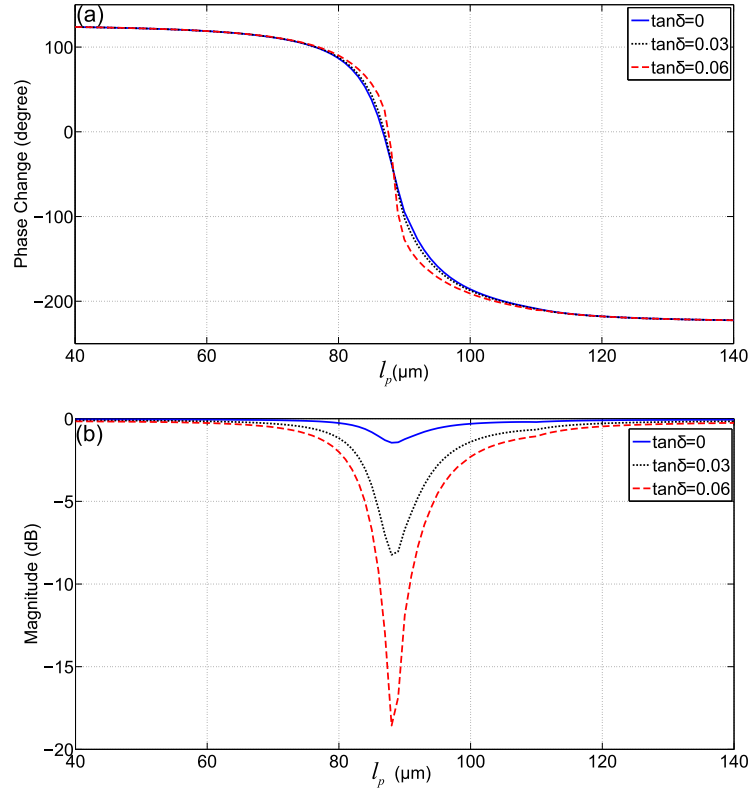

Fig. 2. Reflection phase change in degree (a) and magnitude in $\mathrm{dB}$ (b) for the structure operating at $1 \mathrm{THz}$ shown in Fig. 1 as a function of the length $l_{p}$, for a dimension $a=150 \mu \mathrm{m}, h=20 \mu \mathrm{m}$. For a flat phase curve, the width $w_{p}$ varies with the length $l_{p}$ according to the relation $w_{p}=32 \mu \mathrm{m}+$ $0.2 \times l_{p}$ when $40 \mu \mathrm{m} \leq l_{p} \leq 90 \mu \mathrm{m}, w_{p}=230 \mu \mathrm{m}-2 \times l_{p}$ when $90 \mu \mathrm{m}$ $\leq l_{1} \leq 105 \mu \mathrm{m}, w_{p}=20 \mu \mathrm{m}$ when $105 \mu \mathrm{m} \leq l_{p} \leq 140 \mu \mathrm{m}$. The loss tangent of the PDMS substrate affects both the phase and magnitude responses.

\section{DESIGN OF DUAL-DEFLECTION REFLECTARRAY}

Based on the phase response data shown in Fig. 2(a), a subarray containing two groups of 6 dipoles is designed for deflecting an incident wave into two different directions according to the polarization components. For simplicity of the theoretical design, we assume that the substrate is lossless, and therefore the blue solid line in Fig. 2 (a) is used for designing the subarray. At $1 \mathrm{THz}$, i.e. $\lambda_{0}=300 \mu \mathrm{m}$, a progressive phase change of $\Delta \phi= \pm 60^{\circ}$ between adjacent dipoles with the same alignment results in a beam deflection of

$$
\theta=\arcsin \frac{\Delta \phi \lambda_{0}}{2 \pi a}= \pm 19.5^{\circ}
$$

The layout of the subarray is shown in Fig. 3. Taking the fabrication tolerance into account, the micrometer values for the length and the width of each dipole in the subarray are chosen as integers. In order to achieve more accurate phase response of each dipole thus better performance of the reflectarray, the phase of the surface current on the dipoles are observed during the subarray simulations. Both of the lengths and widths of the dipoles in the subarray are then fine-tuned to achieve the desired gradient phase response. The optimized dimensions of the 12 dipoles are given in Table I.

In designing the reflectarray, possible coupling effects between adjacent dipoles of different orientations have to be taken into account. One property that indicates this aspect is the distribution of the surface current on the strip dipoles. So, it is necessary to observe the distributions of the surface current on the dipoles, as shown in Fig. 4 for both the TE and TM incident polarizations (note that TE and TM are defined in the

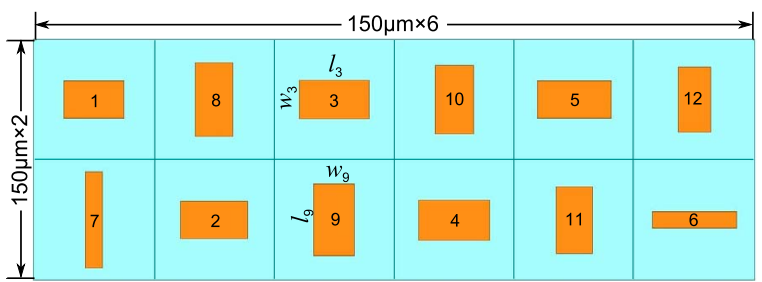

Fig. 3. Structure of one subarray made of 12 dipoles. The figure is to scale.

TABLE I. SIZES OF THE DIPOLES FOR THE DESIGNED SUBARRAY. THE UNITS ARE IN $\mu m$.

\begin{tabular}{|c|cccccc|}
\hline & 1 & 2 & 3 & 4 & 5 & 6 \\
\hline$l_{p}$ & 75 & 84 & 87 & 89 & 92 & 105 \\
$w_{p}$ & 47 & 47 & 48 & 50 & 47 & 21 \\
\hline & 7 & 8 & 9 & 10 & 11 & 12 \\
\hline$l_{s}$ & 120 & 92 & 89 & 85 & 83 & 81 \\
$w_{s}$ & 21 & 47 & 51 & 48 & 45 & 40 \\
\hline
\end{tabular}

deflection plane). It is clear that the surface currents on the dipoles responding to one specific polarization are dominant compared to the other polarization. This further verifies that the effect of the dipoles is negligible for the incident wave with orthogonal polarization.

The simulated incident plane wave at $1 \mathrm{THz}$ shown in Fig. 5(b) is normal to the surface of the reflectarray. The field distributions of the deflected waves for the TE and TM polarizations are demonstrated in Figs. 5(a) and (c), respectively. It is clear that the normal incident wave is deflected into the predesigned direction depending on the polarization. For the TM polarization, the deflected wavefront is dominant over the specular reflection component in the scattered field. On the other hand, for the TE polarization, an obvious specular reflection component is observed. The phenomenon has also been observed and discussed by Niu et al. [17]. Overall, the results confirm that the designed reflectarray is able to deflect terahertz waves into the two designed different directions, according to the incident polarization.

For further evaluation of the performance of the designed reflectarray, we consider a reflectarray that contains $168 \times 56$ subarrays. This corresponds to a sample with a size of $50.4 \mathrm{~mm} \times 50.4 \mathrm{~mm}$, which is sufficient to cover the colli-

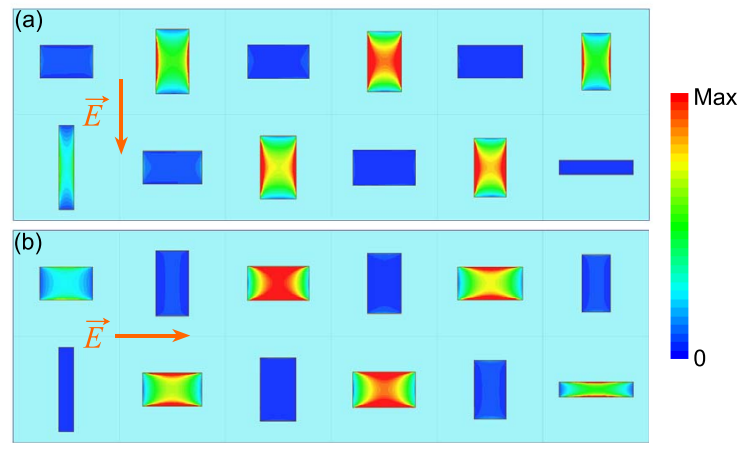

Fig. 4. Magnitude of the surface current on the dipoles responding to the TE (a) and TM (b) polarizations. The both images share the same color scale. 


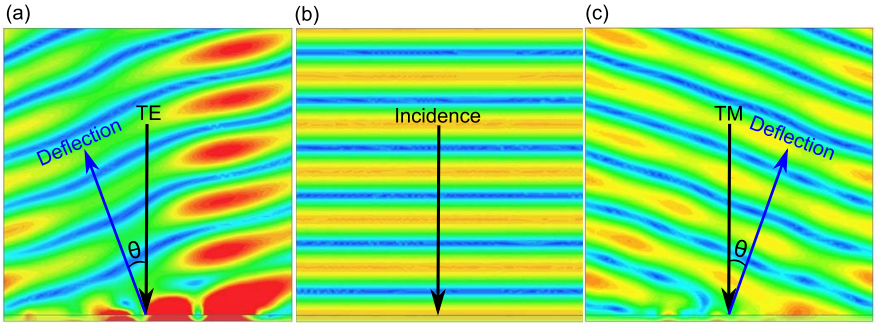

Fig. 5. Instantaneous scattered field from the reflectarray in TE and TM polarizations at $1 \mathrm{THz}$. When the incident wave (b) is normal to the surface of the reflectarray, the TE and TM polarized wave are deflected into two different directions with the angles of $\pm 19.5^{\circ}$ as shown in (a) and (c), respectively. All the images share the same color scale.

mated beam in the measurement. Based on this configuration, the radiation pattern can be numerically obtained (neglecting edge effects) by using Ansys HFSS commercial software as shown in Fig. 6. It is obvious that the incident waves with the TE and TM polarizations can be efficiently deflected into two different directions. For the TM polarization, the intensity in the designed deflection direction is around $20 \mathrm{~dB}$ higher than that in the specular reflection, while for the TE polarization the difference is smaller. The results further confirm that the designed reflectarray can separate the polarization components of an incident beam by deflecting them into two different directions in a given plane.
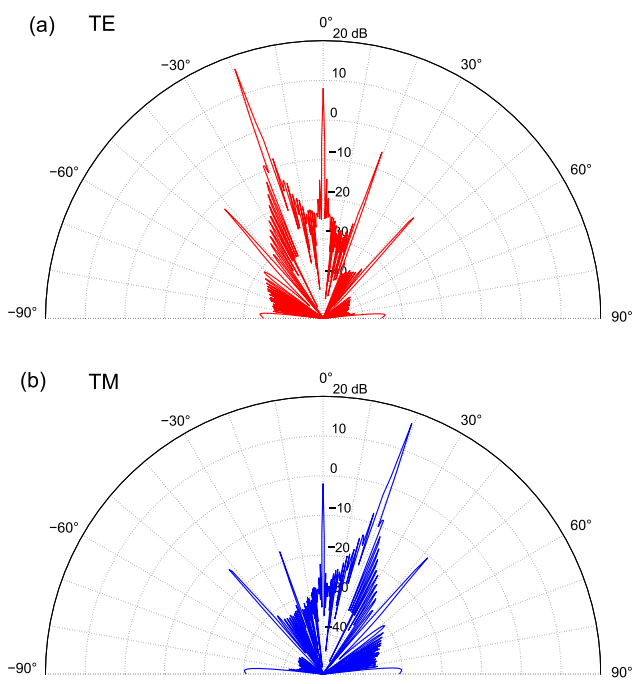

Fig. 6. Radiation pattern of the reflectarray with a dimension of $50.4 \mathrm{~mm} \times 50.4 \mathrm{~mm}$. A wave for normal incidence at $0^{\circ}$ with TE and TM polarization can be efficiently deflected onto the direction of $-19.5^{\circ}$ (a) or $+19.5^{\circ}$ (b), respectively.

\section{CONCLUSION}

A terahertz reflectarray for polarization-dependent deflection has been designed and simulated. The simulation results verify that the reflectarray can deflect the incident waves with different polarizations into desired directions. It is noted that, to avoid high loss and sharp phase response, the substrate adopted in the design is lossless, which is not realistic. One approach to solve this issue is to improve the layout of the strip dipoles on the top layer for a flatter phase curve and lower loss. The related work and experimental validation are in progress, and the prototype can be measured through terahertz time-domain spectroscopy (THz-TDS) used in [17].

\section{ACKNOWLEDGMENT}

Christophe Fumeaux acknowledges the ARC Future Fellowship funding scheme under FT100100585.

\section{REFERENCES}

[1] D. M. Pozar, S. D. Targonski, and H. D. Syrigos, "Design of millimeter wave microstrip reflectarrays," IEEE Trans. Antennas Propag. 45, 287296, 1997.

[2] M. Farmahini-Farahani and H. Mosallaei, "Birefringent reflectarray metasurface for beam engineering in infrared," Opt. Letts. 38, 462-464, 2013.

[3] J. Ginn, B. Lail, J. Alda, and G. Boreman,"Planar infrared binary phase reflectarray," Opt. Lett. 33, 779-781, 2008.

[4] A. Pors, O. Albrektsen, I. P. Radko, and S. I. Bozhevolnyi, "Gap plasmon-based metasurfaces for total control of reflected light," Scientific Reports 3, 2155, 2013.

[5] L. Zou, W. Withayachumnankul, C. M. Shah, A. Mitchell, M. Bhaskaran, S. Sriram, and C. Fumeaux, "Dielectric resonator nanoantennas at visible frequencies," Opt. Express 21, 1344-1352, 2013.

[6] J. Huang and J. Encinar, Reflectarray Antenna. Wiley Online Library, 2008.

[7] N. Grady, J. Heyes, D. Chowdhury, Y. Zeng, M. Reiten, A. Azad, A. Taylor, D. Dalvit, and H. Chen, "Terahertz metamaterials for linear polarization conversion and anomalous refraction," Science 340, 13041307, 2013.

[8] X. Zhang, Z. Tian, W. Yue, J. Gu, S. Zhang, J. Han, and W. Zhang, "Broadband terahertz wave deflection based on C-shape complex metamaterials with phase discontinuities," Adv. Mater. 25, 4567-4572, 2013.

[9] J. Encinar, M. Arrebola, L. F. de la Fuente, and G. Toso, "A transmitreceive reflectarray antenna for direct broadcast satellite applications," IEEE Trans. Antennas Propag. 59, 3255-3264, 2011.

[10] E. Carrasco, M. Tamagnone, and J. Perruisseau-Carrier, "Tunable graphene reflective cells for $\mathrm{THz}$ reflectarrays and generalized law of reflection," Appl. Phys. Lett. 102, 104103, 2013.

[11] N. Vieweg, M. K. Shakfa, B. Scherger, M. Mikulics, and M. Koch, "THz properties of nematic liquid crystals," J. Infr. Millim. Terahertz Waves, 31, 1312-1320, 2010.

[12] M. Y. Ismail, W. Hu, R. Cahill, V. Fusco, H. S. Gamble, D. Linton, R. Dickie, S. P. Rea, and N. Grant, "Phase agile reflectarray cell based on liquid crystals," IET Microw. Antennas Propag., 1, 809-814, 2007.

[13] G. Perez-Palomino, P. Baine, R. Dickie, M. Bain, J. Encinar, R. Cahill, M. Barba, and G. Toso, "Design and experimental validation of liquid crystal-based reconfigurable reflectarray elements with improved bandwidth in F-band", IEEE Trans. Antennas Propag. 61, 1704-1713, 2013.

[14] J. Encinar, L. Datashvili, and J. Zornoza, M. Arrebola, M. SierraCastañer, and J. Besada-Sanmartín, H. Baier, H. Legay, Herve, "Dualpolarization dual-coverage reflectarray for space applications," IEEE Trans. Antennas Propag. 54, 2827-2837, 2006.

[15] J. Perruisseau-Carrier, "Dual-polarized and polarization-flexible reflective cells with dynamic phase control," IEEE Trans. Antennas Propag. 58, 1494-1502, 2010.

[16] W. Withayachumnankul, C. Fumeaux, and D. Abbott,"Near-field interactions in electric inductive-capacitive resonators for metamaterials," $J$. Phys. D: Appl. Phys. 45, 485101, 2012.

[17] T. Niu, W. Withayachumnankul, B. S.-Y. Ung, H. Menekse, M. Bhaskaran, S. Sriram, and C. Fumeaux ,"Experimental demonstration of reflectarray antennas at terahertz frequencies," Opt. Express 21, 28752889, 2013.

[18] I. E. Khodasevych, C. M. Shah, S. Sriram, M. Bhaskaran, W. Withayachumnankul, B. S. Y. Ung, H. Lin, W. S. T. Rowe, D. Abbott, and A. Mitchell, "Elastomeric silicone substrates for terahertz fishnet metamaterials,” Appl. Phy. Lett. 100, 061101, 2012. 